\title{
Langue de spécialité et noms propres : comparaison des noms de matériels militaires britanniques et américains
}

Jean-Louis Trouillon

\section{(2) OpenEdition \\ Journals}

Édition électronique

URL : http://journals.openedition.org/asp/2827

DOI : 10.4000/asp.2827

ISSN : 2108-6354

Éditeur

Groupe d'étude et de recherche en anglais de spécialité

\section{Édition imprimée}

Date de publication : 1 décembre 1998

Pagination : 209-225

ISSN : 1246-8185

\section{Référence électronique}

Jean-Louis Trouillon, «Langue de spécialité et noms propres : comparaison des noms de matériels militaires britanniques et américains », ASp [En ligne], 19-22 | 1998, mis en ligne le 15 mars 2012, consulté le 19 avril 2019. URL : http://journals.openedition.org/asp/2827 ; DOI : 10.4000/asp.2827

Ce document a été généré automatiquement le 19 avril 2019

Tous droits réservés 


\title{
Langue de spécialité et noms propres: comparaison des noms de matériels militaires britanniques et américains
}

\author{
Jean-Louis Trouillon
}

TOW stands for tube-launched, optically-tracked,
wire-guided. As the acronym suggests, TOW is
American in origin, being one of the main products
of Hugues Missile Systems. (Gander $1995: 108$ )

1 Cet extrait d'un ouvrage britannique nous invite à nous interroger sur la spécificité supposée des noms de matériels militaires américains (US) par rapport aux appellations données par les Britanniques (UK) pour le même type de produits. Est-il possible d'envisager, tout au moins au niveau lexical et dans ce domaine particulier, une notion d'ethnicité dans le cadre d'une langue spécialisée ? L'étude portera donc sur l'analyse des critères qui sous-tendent l'attribution d'un nom à un matériel dans un contexte militaire. Pour Handwerker en effet

[...] les terminologies militaires diffèrent largement de leurs homologues dans d'autres disciplines quant à leurs intérêts de motivation. (1984:29)

2 Suite à l'étude menée sur l'Armée de Terre française par Marie-Anne Pavaud (1994) les outils d'analyse retenus relèveront plus particulièrement de l'ethnolinguistique. Il sera également fait appel aux travaux menés en sciences de gestion sur les noms de marques et de produits.

\section{Cadre du sujet}

3 Afin de restreindre le champ de l'étude, et compte tenu de la documentation accessible, l'analyse porte sur les matériels contemporains de fabrication nationale en dotation au sein des Armées de Terre britannique et américaine, à l'exclusion donc de la Marine et de l'Armée de l'Air. Par contemporain, nous entendrons depuis la fin de la Deuxième Guerre 
mondiale à nos jours, y compris les matériels les plus récents en cours d'introduction au sein des forces (courant 1998). Le corpus est donc fini à la date de l'étude et facilement analysable dans son intégralité.

Les matériels américains sont habituellement désignés au départ par un code alphanumérique utilisant généralement la lettre $\mathrm{M}$ (ex. : M109 où $M$ est l'initiale de Model et 109 représente simplement un ordre chronologique d'étude ou de fabrication).

Les matériels britanniques obéissent au même principe alphanumérique mais les lettres sont plus variées et correspondent aux initiales d'un syntagme nominal (SN). Par exemple, AT2 est une mine anti-char (AT = Anti-Tank), FV432 est un véhicule de combat (FV = Fighting Vehicle), etc. La référence numérique peut être aussi bien un ordre de production qu'une référence chronologique. Ex.: dans AS90, AS signifie Artillery Selfpropelled et 90 représente for the 90 s.

6 Une évolution de matériel n'entraînera généralement, et ce aussi bien chez les Américains que chez les Britanniques, que l'ajout d'un élément caractérisant la chronologie : c'est ainsi que Abrams M1 est devenu Abrams M1A1 et que Challenger 2 est le successeur naturel de Challenger 1.

7 Parallèlement, un certain nombre de ces matériels, aussi bien UK que US, sont également baptisés ou se voient ultérieurement affublés d'un surnom. Parmi les catégories de noms de baptême originaux, il faut notamment citer :

- un SN dénotatif de la fonction du produit ; ex. : Rough Terrain Fork-lift Truck (UK)

- un sigle, abréviation d'un SN ; ex. : HMMWV abréviation de High Mobility Multipurpose Wheeled Vehicle (US)

- un nom propre (Npr), anthroponyme, théonyme, toponyme ou ethnonyme ; ex. : Abrams (US), Titan (US), Chobham (UK), Apache (US)

- un nom commun $(\mathrm{Nc})$ devenant nouveau signifiant pour un signifié différent par apparition d'une majuscule à l'initiale; ex. : Sabre (UK)

- un Nc formé par suffixation selon les règles habituelles de la langue anglaise ; ex. : Breacher (US)

- un Nc formé par amalgame ; ex. : Stillbrew

8 Les codes originaux ne seront pas étudiés ici car relevant d'un simple processus de classification propre aux commanditaires et constructeurs. Ils pourront toutefois être mentionnés lorsque, du fait de leur coexistence avec un nom, la présence d'un double signifiant pour un même signifié sera perçue comme pertinente. Les SN non abrégés ne seront pas non plus étudiés.

Pour le spécialiste du marketing,

loin d'être le fruit d'une réflexion hasardeuse, le nom désignant la marque constitue un élément essentiel de la stratégie. En général, il est préférable qu'un nom de marque possède l'une ou l'autre des qualités suivantes :

- il doit évoquer les avantages procurés par l'utilisation du produit: ronron, taillefine

- il doit suggérer des attributs du produit tels que l'aspect, la composition, la couleur : mousline

- il doit être facile à prononcer, à reconnaître et à mémoriser. Les noms courts sont à cet égard préférables : bic, omo

- il doit être distinctif : kodak

- il ne doit pas être associé à des connotations négatives dans d'autres langues

Certaines sociétés ont mis au point des méthodes élaborées de recherche de noms : les plus utilisées sont les tests d'association (quelles images viennent à l'esprit?), 
les tests d'élocution (la prononciation est-elle aisée ?), les tests de mémorisation (est-il facile de se souvenir du nom ?) et les tests de préférence (quels noms sont préférés ?) (Kotler \& Dubois 1994 : 447)

Pour le linguiste,

Les organismes, les entreprises, les associations nouveaux, etc. essaient de trouver des sigles parlants et efficaces qui s'enregistrent vite et se retiennent facilement. On a tendance à rechercher de plus en plus des sigles directement prononçables dont le signifiant est parfois homonyme d'un mot existant. (Niklas-Salminen 1997 : 82).

On retrouvera a priori des éléments de convergence entre les noms de matériels étudiés et les critères énoncés ci-dessus :

10 - par dénotation: les $\mathrm{SN}$ définissant les produits évoquent systématiquement leur fonction (Ex. : Automotive Bridge Launching Equipment).

11 - par connotation : pour ce qui relève de l'association et de la mémorisation on peut noter qu'au sein de l'actuelle Série $S$ de véhicules britanniques présentant une déclinaison de modèles (Sabre, Samaritan, Samson, Scorpion, Spartan, Stormer, Sultan, Scimitar, Striker) construits à partir d'un châssis commun, seuls Sabre, Scorpion, Scimitar et Stormer ne se prêtent à aucun procédé mnémotechnique évident quant à la destination du véhicule. En effet, Samson est le véhicule de remorquage capable de tirer de lourdes charges, Sultan désigne le véhicule de commandement, Striker est chargé de porter des coups à l'ennemi en détruisant ses chars au moyen de missiles. Quant à Samaritan, le nom désigne le véhicule ambulance. Dans ce dernier cas, les parrains ont reproduit la synecdoque d'origine populaire qui semble dans l'imaginaire collectif associer en un anthroponyme unique l'ensemble des habitants de la Samarie au Bon Samaritain de la Bible.

La motivation est moindre pour les autres véhicules de la série : même si Scorpion évoque une idée de létalité rien ne permet de déterminer que ce véhicule a reçu la même mission que Striker mais avec un canon et non un missile. Sabre, Scimitar et Stormer possèdent certes des connotations guerrières, mais la finalité exacte du véhicule reste inconnue.

\section{L'emprunt}

\subsection{Noms communs (Nc)}

\section{Zoonymes}

Une étude des fréquences d'occurrence totale (UK+US) permet de classer en tête les noms d'animaux qui jouent sans aucun doute une fonction totémique (Pavaud 1994:473) à laquelle les Britanniques font plus largement appel que les Américains (15 occurrences contre 6):

UK : Bloodhound, Dragon' Ferret, Fox, Giant Viper, Kite, Lynx, Phoenix, Ptarmigan, Python, Rhino, Scorpion, Terrier, Tigercat, Wombat US : Bushmaster, Cobra, Copperhead, Grizzly, Redeye, Wolverine

Plusieurs tentatives de classification sont possibles (voir tableau 1).

Tableau 1. Classification de type taxonomie biologique (règne animal uniquement)

\begin{tabular}{|l|l|l|l|l|l|}
\hline & Arachnide & Mammifère & Oiseau & Poisson & Reptile \\
\hline
\end{tabular}




\begin{tabular}{|l|l|l|l|l|l|l|}
\hline UK & 1 & 8 & 3 & 0 & 3 \\
\hline & US & 0 & 2 & 0 & 1 & 3 \\
\hline & Total & 1 & 10 & 3 & 1 & 6 \\
\hline
\end{tabular}

Le dragon a été classé parmi les reptiles. On ne trouve ni mollusque ni insecte. Les Britanniques semblent privilégier les mammifères qui à eux seuls dépassent en valeur absolue le total des quatre autres catégories.

Tableau 2. Classification de type autochtone/allochtone

\begin{tabular}{|l|l|l|l|}
\hline & Autochtone & Allochtone \\
\hline & UK & 12 & 3 \\
\hline & US & 6 & 1 \\
\hline & Total & 18 & 4 \\
\hline
\end{tabular}

Le dragon et le phénix ont été classés parmi les autochtones. Dans les deux cas, les autochtones sont préférés aux allochtones.

Tableau 3. Classification de type réel / fabuleux

\begin{tabular}{|l|l|l|l|}
\hline & Réel & Fabuleux \\
\hline & UK & 13 & 2 \\
\hline & US & 7 & 0 \\
\hline & Total & 20 & 2 \\
\hline
\end{tabular}

L'absence totale de référents fabuleux chez les Américains pourrait s'expliquer aussi bien par une impossibilité historique de référence à un passé moyenâgeux que par un souci de réalisme...

Ces classifications ne présentent toutefois qu'un intérêt anecdotique. Il semble plus pertinent de chercher dans les noms retenus des notions porteuses de sens aux yeux de la communauté militaire. C'est ainsi que l'on peut retenir les concepts de prédation, de force, de ruse, de vivacité, auxquels il est possible, grâce à Phoenix (UK), de rajouter l'idée d'éternité et donc d'invincibilité face à la défaite ou à la mort. Les auteurs font donc appel à une "stratégie de domaine » dans leur quête d'appellation (Durafour $1993: 25$ ).

Le cas de Ptarmigan (UK) apparaît comme totalement non motivé, aucune des caractéristiques du volatile en question ne le prédisposant a priori à un emprunt par les forces armées de Sa Majesté pour baptiser un système de communication.

Noms de guerriers 
Au deuxième rang par ordre décroissant d'occurrence, on constate la présence de noms de guerriers ou tout au moins à connotation mixte :

UK : Centurion, Challenger, Chieftain, Conqueror, Striker, Sultan

US : Avenger, Hunter, Paladin, Patriot, Sergeant

Une répartition de type historique donne le résultat figurant au tableau 3.

Tableau 4. Répartition de type historique

\begin{tabular}{|c|c|c|c|c|}
\hline & Antiquité & Moyen-Âge & Allochtone & Non marqué \\
\hline UK & 1 & 1 & 1 & 3 \\
\hline US & 0 & 1 & 0 & 4 \\
\hline Total & 1 & 2 & 1 & 7 \\
\hline
\end{tabular}

22 Aucune différence notable à ce niveau compte tenu du petit nombre d'éléments considérés.

\section{Armes anciennes}

23 Au troisième rang, on trouve en nombre à peu près égal (quatre contre cinq) des noms d'armes anciennes :

UK : Blowpipe, Javelin, Sabre, Scimitar

US : Claymore, Javelin, Lance, Longbow, Rapier

Tableau 5. Noms d'armes anciennes

\begin{tabular}{|l|l|l|l|}
\hline & Autochtone & Allochtone \\
\hline & UK & 2 & 2 \\
\hline US & 0 & 5 \\
\hline & Total & 2 & 7 \\
\hline
\end{tabular}

24 Si chez les Britanniques des noms locaux font jeu égal avec des noms plus exotiques, les Américains ne font appel qu'à leurs sources européennes ${ }^{1}$.

Il est à noter l'existence exceptionnelle d'un même signifiant Javelin pour deux systèmes d'armes différents (arme UK sol air, arme US antichar). Cette identité est d'autant plus surprenante qu'elle se trouve au sein de deux communautés partageant, pour des raisons historiques, linguistiques, économiques et politiques, de nombreux concepts communs. On se serait donc attendu à vérifier le principe de monoréférentialité énoncé par Pottier (1992: 65).

\section{Références religieuses chrétiennes : hommes d'Église}


Une tradition récemment éteinte de la Royal Artillery a longtemps voulu que les pièces d'artillerie portent des titres d'hommes d'église : c'est ainsi que Priest et Cardinal ont laissé la place à Abbot en usage jusqu'à l'introduction récente du AS90 pour lequel aucun nom n'a été proposé. L'idée de puissance divine, «the wrath of God » précédemment impliquée semble donc avoir disparu au profit d'une froide réalité (cf. supra).

Le phénomène religieux chrétien était, et est toujours, inexistant chez les Américains. Il est néanmoins curieux de constater en artillerie une inversion des habitudes de dénomination : alors que les Britanniques remplacent leur Abbot par un AS90, la dernière version de l'américain M109 est un Paladin!

\section{Phénomènes naturels}

Un unique nom (US) de phénomène physique naturel, Volcano, avec connotation de puissance irrésistible.

\section{Sportifs}

En tout dernier lieu, l'emprunt d'un SN destiné à préciser une variante du véhicule Bradley (US) : Linebacker.

L'exemple est doublement intéressant en ce sens qu'il peut s'agir là aussi bien d'un emprunt direct au registre du football américain que d'un emprunt décalé, Linebacker ayant déjà été utilisé, mais dans un sens tactique et non pour un matériel, lors de la Guerre du Vietnam. Si cette deuxième hypothèse est la bonne, nous aurions alors affaire à une double référence avec phénomène de masquage particulièrement subtil puisque la référence de type ésotérique réservée à un tout petit groupe d'initiés au sein même de la communauté se cacherait derrière une première métaphore transparente.

\subsection{Noms propres (Npr)}

Aucun hasard ne saurait déterminer ici les choix :

Les noms propres [...] réfèrent aux individus qui portent ces noms, mais en outre ils évoquent des propriétés liées à ces individus et qui contribuent à l'interprétation sémantique de l'énoncé. (Gary-Prieur 1994 : 12)

Cette remarque s'appliquera donc aux emprunts de Npr puisqu'il y aura ici nécessairement appel volontaire à un référent. Le propos pourra en ce sens dépasser largement le cadre de l'anthroponyme ou théonyme et porter sur l'ethnonyme et le toponyme. On ne s'opposera pas malgré tout à la thèse de Kleiber pour qui :

le risque est grand de charger le Npr de traits sémantiques qui ne sont que des traits factuels du porteur du nom, et de voir dans le sens du Npr tout ou partie de ce que nous savons du porteur du nom. (1994:13)

Le fait que nous ayons affaire à des Npr empruntés sciemment ne saurait en effet infirmer un propos par ailleurs axé sur les patronymes.

Ethnonymes

$\mathrm{Au}$ premier rang par ordre total d'occurrence se trouvent les ethnonymes faisant référence à l'histoire nationale, biblique ou de l'antiquité grecque :

UK : Samaritan, Saracen, Saxon, Spartan, Trojan

US: Apache, Blackhawk, Cayuse, Chinook, Comanche, Iroquois, Kiowa,

Mescalero, Mohawk, Osage, Seminole, Tarhe, Ute 

directement un matériel portant le nom de son inventeur ${ }^{2}$ le culte des grands anciens inauguré pendant la Seconde Guerre dans la Cavalerie US avec les chars Chaffee, General Grant et Sheridan puis Sherman et Patton (encore en dotation au lendemain de la guerre et donc cités dans le cadre de cette étude) se continue de nos jours avec le char Abrams et le véhicule blindé Bradley. Les noms de baptême sont ceux de chefs de l'arme blindée récemment disparus. Le seul contre-exemple est Pershing qui désigne un missile. Ce « culte des ancêtres du groupe » a déjà été remarqué (Pavaud 1994 : 473). Le phénomène est à l'heure actuelle presque totalement absent chez les Britanniques ${ }^{3}$ à l'exception de Stillbrew, NPr formé par troncation et juxtaposition du nom des deux inventeurs du blindage portant ce nom.

On peut rajouter à ce paragraphe Honest John (US) et Little John (US) dont l'existence pourrait se justifier par création paradigmatique à la suite de Long Tom (US), nom métaphorique d'un canon de marine (précédant notre période) dont la forme rappelait l'instrument de travail des orpailleurs du XIX ${ }^{e}$ siècle.

\section{Toponymes}

Enfin, un seul véritable toponyme, Chobham (UK), désignant un type de blindage pour chars: cette localité du Surrey abrite le laboratoire R\&D ayant donné naissance au matériau constituant le blindage. 
41 Un missile sol-air US a été baptisé Chaparral: ce phytonyme désignant un type de végétation localisé dans l'espace américain nous l'avons classé dans la catégorie des toponymes.

\section{Emprunt à une langue populaire}

Un exemple unique avec SUPACAT (UK). Ce "Super Cat », petit véhicule passe-partout hautement mobile, emprunte aussi bien au mythe moderne du Super Héros qu'à la transcription familière de la prononciation de super.

\subsection{Coexistence de signifiants}

Un phénomène de coexistence du code d'origine et du nom de baptême, c'est-à-dire de l'existence de deux signifiants pour un même signifié, mérite d'être signalé à ce niveau. L'exemple le plus courant est celui des aéronefs US pour lequel on pourra même signaler la coexistence de trois signifiants par apparition d'un surnom donné a posteriori par les utilisateurs eux-mêmes et se rajoutant aux dénominations d'origine.

Tableau 7.Coexistence du code d'origine, du nom de baptême et du surnom

\begin{tabular}{|l|l|l|l|l|}
\hline FONCTION & CODE & NOM & SURNOM \\
\hline Attack Helicopter & AH-64 & Apache & \\
\hline Attack Helicopter & AH-1 & Cobra & \\
\hline Cargo Helicopter & CH-47 & Chinook & \\
\hline Cargo Helicopter & CH-54 & Tarhe & Skycrane \\
\hline Observation Helicopter & OH-6 & Cayuse & \\
\hline Observation Helicopter & OH-58 & Kiowa & \\
\hline Observation Plane & OV-1 & Mohawk & \\
\hline Reconnaissance and Attack Helicopter & RAH-66 & Comanche & \\
\hline Training Helicopter & TH-55 & Osage & \\
\hline Training Plane & T-41 & Mescalero & \\
\hline Training Plane & T-42 & Cochise & \\
\hline Utility Helicopter & UH-1 & Iroquois & Huey \\
\hline Utility Helicopter & UH-60 & Blackhawk & \\
\hline Utility Plane & U-21 & Ute & \\
\hline Utility Plane & Seminole & \\
\hline
\end{tabular}



primitive, recoupe la tendance à la connotation métaphorique particulière déjà exprimée, à savoir la plupart du temps la notion de prédation (COBRA), mais aussi celles de capacité ( $A B L E)$ ou encore de qualité et de perfection (ACE) renforçant l'idée de dangerosité du groupe utilisateur par rapport à l'ennemi potentiel. S'il perd en dénotation, c'est-à-dire s'il n'est plus explicite des fonctions premières du matériel, détection, franchissement ou 
terrassement pour reprendre les trois exemples cités (COBRA est un radar ${ }^{5}$, ABLE est un système poseur de ponts et $A C E$ un engin de terrassement), le signifiant retenu gagne en maniabilité (Handwerker 1984 : 56).

Il peut cependant arriver que l'acronyme conserve partiellement la dénotation d'origine : DROPS (UK) a par exemple été retenu pour un système logistique de transport et transfert qui a donc, entre autres fonctions, celle de déposer du matériel.

Cette coïncidence avec un lexème se trouve également parfois hors connotation spécifique. L'exemple de TOW, point de départ de notre étude, mérite d'être signalé. En effet, on assiste ici à une conversion d'ordre syntaxique puisque le sigle, abréviation d'un adjectif déterminant le nom missile, change de catégorie, devenant ainsi substantif par troncation définitive du déterminé :

$$
\text { Ex. : « The Lynx carries up to four TOWs. » (Gander } 1995 \text { : 108) }
$$

53 Cette connotation externe, c'est-à-dire non marquée de militarité, voit parfois la prédominance de la recherche du lexème au détriment de la fonction: c'est le cas de CHARM (UK) ou encore de NAIAD (UK). La visée initiale ayant présidé à l'attribution du SN est donc à remarquer aussi bien au niveau de la dénotation (mais cela n'est valable que pour le SN d'origine dont on sait bien qu'il va s'effacer) que de la recherche de maniabilité pour l'utilisateur. Les arrières pensées commerciales ne sont sans doute pas absentes lors d'un tel choix. "Un producteur, en baptisant son produit, voudra susciter des associations agréables chez le futur acheteur. » (Jonasson 1994 : 123).

Malgré de tels efforts de créativité, il arrive que la communauté militaire néglige le sigle : dans le discours oral quotidien, pour désigner le véhicule Truck Utility Medium, c'est-à-dire TUM, les Britanniques parlent simplement de Land Rover!

Au plan morphologique, on peut citer le cas particulier de Joint STARS (US) qui accole en un SN d'apparence poétique l'adjectif joint (c'est-à-dire interarmées, le radar en question étant également en service dans la marine et l'Armée de l'Air) et le sigle STARS ( Surveillance Target Attack Radar System) ${ }^{6}$.

\subsubsection{Acronymes lexicalisés}

L'abondance des sigles non lexicalisés ou non lexicalisables est telle qu'ils ne seront pas traités au sein de cette étude.

On peut néanmoins citer ici l'exemple d'un lexème obtenu par un processus inverse de lexicalisation: HMMWV devient Humvee. Le même phénomène s'était produit pour la première fois lors de la Deuxième Guerre mondiale, donc dans une phase antérieure à l'époque retenue pour notre étude. Il s'agissait de la célèbre Jeep : General Purpose Wheeled Vehicle d'abord abrégé en GPW Vehicle, GP devenant enfin phonétiquement JEEP.

Dans ces deux cas, il ne s'agit pas exactement de noms de baptême originellement donnés par les constructeurs mais d'une sorte de baptême a posteriori administré par les utilisateurs eux-mêmes, avec effacement systématique du sigle initial dans le cas de la JEEP. Pour ce qui est du Humvee, si le sigle HMMWV demeure le seul admis dans les documents écrits officiels, le nom Humvee est de mise dans tous les autres cas d'occurrence. On retrouve sans doute ici un besoin d'appropriation dû à la catégorie : véhicule de base, proche d'une automobile «civile» que tout utilisateur tend naturellement à personnaliser. 


\subsubsection{Acronymes lexicalisables}

On relève peu d'exemples d'acronymes lexicalisables. Le phénomène est d'ailleurs quelque peu subjectif : MICLIC, bien qu'obéissant aux règles lexicogéniques de la langue, peut-il être considéré comme lexicalisable?

Deux d'entre eux se rapprochent d'onomatopées, ce que sous la dénomination « néologie phonologique spécifique » Guilbert (1975: 60) définissait comme «articulation d'une ou plusieurs syllabes qui aient une valeur signifiante inédite ». Il s'agit de HESH et HETS. Cette relative pauvreté est probablement à expliquer par la forte présence des autres catégories, l'acronyme lexicalisable étant sans doute considéré comme un pis-aller. Tout au plus peut-on mentionner la richesse de HESH (High Explosive Squash Head) qui réussit à conserver le phonème $\left[\begin{array}{c}{\left[\mathrm{F}_{2}\right]} \\ \mathrm{F}\end{array}\right]$ également présent dans squash.

\subsection{Les surnoms}

1 Ils restent rares, même s'il s'agit d'une tradition ancienne (cf. les chars Matilda dans l'armée britannique). On trouve toutefois, et en nombre à peu près égal dans les deux communautés :

Pig et Piglet, surnoms donnés à certaines versions de Land Rover utilisées par l'armée britannique pour le maintien de l'ordre en Ulster.

Pink Panther, surnom donné aux véhicules Land Rover peints en camouflage rose utilisés en milieu désertique par le Special Air Service (SAS).

Mechanical MULE pour le M274 (US), sorte de Jeep de la fin des années 50.

Deux hélicoptères: le $\mathrm{CH}-54$ Tarhe est souvent appelé Skycrane en raison de ses exceptionnelles capacités d'emport de matériel, et le UH-1 Iroquois a toujours été connu sous le nom de Huey: on se rapproche dans ce dernier cas du phénomène de création phonétique déjà évoqué pour Jeep et Humvee.

\subsection{Troncation}

Si l'on excepte les SN mentionnés ci-dessus (JEEP, TOW) ainsi que Stillbrew, on ne relève aucun exemple de troncation créatrice : Champ (UK) est en effet attesté dès 1868 et Rhino (UK) à partir de 1884 (pour le signifié mammifère).

L'exemple de HELLFIRE (US), Helicopter Launched Fire and Forget Missile, mérite d'être signalé de par son processus de formation complexe puisqu'il mêle troncation par apocope et siglaison.

68 Un phénomène identique préside à la naissance de Rarden (UK) : Royal Armament $\mathbf{R}$ esearch and Development Establishment auquel on a rajouté Royal Small Arms Factory at Enfield Lock.

\section{Conclusion}

L'affirmation de Gander (1995: 108) à l'origine de notre étude n'apparaît nullement avérée: même si les quantités sont trop faibles pour que l'on puisse en tirer des 
indications statistiques fiables on peut en effet dire que les acronymes lexèmes se retrouvent en majorité chez les Britanniques.

Il est en revanche possible de dégager deux grandes tendances quant à la dénomination : les Britanniques préfèrent dans l'ensemble les zoonymes, alors que les Américains restent plus attachés aux anthroponymes et ethnonymes.

Il faut toutefois nuancer les remarques proposées : les matériels sont en effet baptisés par des constructeurs plus ou moins indépendants des ministères de la Défense et dont les produits ne sont pas tous systématiquement acquis par les deux armées étudiées : par exemple, l'armée de terre britannique expérimente en ce moment le système SCARAB du constructeur Alvis dont rien ne dit qu'il sera un jour en dotation au sein des forces. Dans ce cas, il n'y aura toujours pas d'insecte parmi les zoonymes observables alors que le nom existera bel et bien.

72 Cette réserve posée, on peut affirmer que les traits présidant à l'attribution des noms relèvent des mêmes principes au sein des deux forces. Les seules différences notables résident dans des tendances, mais il semble bien que cela corresponde à une notion de mode (Cromwell et Churchill ont en effet été un moment utilisés chez les Britanniques lors de la Deuxième Guerre mondiale) et dans la typologie de désignation de séries : tribus indiennes chez les Américains, lettre initiale $\mathbf{S}$ ou $\mathbf{C}$ chez les Britanniques. Il ne s'agit en fait que de simples manifestations de surface d'une même recherche de volonté d'appartenance à une série même si la marque immédiatement observable d'appartenance est différente.

Il apparait donc bien que, contrairement à l'hypothèse un instant soulevée, on ne puisse, au niveau des Npr tout au moins et dans le domaine étudié, introduire un élément d'ethnicité au sein de la langue spécialisée : le concept de spécialité dépasse les frontières nationales.

Les Npr dans l'armée sont à regarder comme des porteurs de l'identité et de

l'existence du groupe, c'est-à-dire des porteurs de militarité (Pavaud 1990 : 465).

Cette phrase écrite à propos de l'armée de terre française semble bien se vérifier pour les armées de terre britannique et américaine.

Je tiens à remercier Monsieur le Général de Brigade Forrer, le Lieutenant Colonel Lee (US Army), le Lieutenant Colonel Martin (British Army), le Lieutenant Colonel Wilson (British Army), Mrs. White (Bovington Tank Museum) qui m'ont aidé à élucider une partie du corpus. Jean-Louis Caccomo m'a guidé dans ma recherche du point de vue du chercheur en sciences de gestion.

\section{BIBLIOGRAPHIE}

Calvet, Louis Jean. 1980. Les sigles. Paris : Presses Universitaires de France.

Durafour, Daniel. 1993. Action commerciale. Paris : Dunod.

Gander, Terry. 1995. Britain's Modern Army. Sparkford : Patrick Stephens Limited. 
Gary-Prieur, Marie-Noëlle. 1994. Grammaire du nom propre. Paris : Presses Universitaires de France.

Guilbert, Louis. 1975. La créativité lexicale. Paris : Larousse.

Handwerker, Bernd. 1984. Les mots de la guerre et la guerre des mots. Francfort : Peter Lang.

Jonasson, Kerstin. 1994. Le nom propre. Constructions et interprétations. Louvain-la-Neuve : Duculot.

Kleiber, Georges. 1994. « Sur la définition des noms propres ». In Noailly, Michèle (dir.), Nom propre et nomination, Actes du colloque de Brest.

Kotler, Philip \& Bernard Dubois. 1994. Marketing management. Paris : Publi-Union Éditions.

Niklas-Salminen, Aïno. 1997. La lexicologie. Paris : Armand Colin.

Pavaud, Marie-Anne. 1994. Le Langage des militaires, Élements pour une ethnolinguistique de l'armée de terre française. Lille : ANRT, Université de Lille 3.

Pottier, Bernard. 1992. Sémantique générale. Paris : Presses Universitaires de France.

Rey, Alain. 1992. La terminologie. Noms et notions.Paris : Presses Universitaires de France.

Bibliographie complémentaire

Botton, Marcel et Jean-Jacques Cegarra. 1990. Le nom de marque. Paris : McGraw Hill.

Williams, Ralph R. 1979. The Language of the Army in English., New York : Regents Publishing Company.

http://www.army.mil/

http://www.army.mod.uk/army/index2.htm

http://www.cfcsc.dnd.ca/links/milorg/gb.html

http://www.cfcsc.dnd.ca/links/milorg/us.html

http://www.m-w.com/netdict.htm

\section{NOTES}

1. Il existe bien un Tomahawk mais il s'agit d'un missile mis en oeuvre par la Marine (US Navy).

2. La première mitrailleuse Gatling a vu le jour en 1861.

3. Des chars Churchill et Cromwell ont néanmoins vu le jour pendant la Seconde Guerre mondiale.

4. Le Breacher porte maintenant le nom de Grizzly

5. Il s'agit bien ici du signifiant COBRA et non de son homophone Cobra déjà évoqué.

6. Ne faut-il pas voir ici en outre un jeu de mots avec les Joint Chiefs of Staff, aréopage d'officiers généraux aux multiples étoiles? 


\section{RÉSUMÉS}

L'analyse d'un corpus fini et fortement motivé, à savoir les noms de matériels militaires contemporains aux États-Unis et en Grande-Bretagne, permet de poser la question d'une éventuelle différence de traitement lexical entre les deux aires linguistiques et en raison du changement éventuel de motivation dans un domaine identique.

Through the analysis of a finite, specific and highly motivated corpus, i.e., the names of contemporary American and British military equipment, the paper examines the possibility of significant lexical differences between a British and American approach to a similar environment.

\section{INDEX}

Mots-clés : armée de terre, connotation, militarité, motivation, nom (propre)

Keywords : army, militarity, name (proper)

\section{AUTEUR}

\section{JEAN-LOUIS TROUILLON}

Jean-Louis Trouillon est maître de conférences à l'Université de Perpignan (IUP de Mende). Outre son intérêt pour le lexique de spécialité, il est l'auteur de nombreux didacticiels. Il fait partie de l'équipe nationale du DEA de langue anglaise de spécialité. jean-louis.trouillon@wanadoo.fr 